excision of the primary tumor while axillary clearance is not routinely indicated and the role of adjuvant procedures, whether chemotherapy or radiotherapy, remains unclear because of limited data.

\section{CONFLICT OF INTEREST STATEMENT}

All authors deny any conflict of interest.

\title{
Breast Sporotrichosis
}

\section{Érica de Camargo Ferreira e Vasconcellos, MSc,* Cláudia Maria Valete- Rosalino, PhD,,${ }^{\dagger \dagger}$ Mônica Bastos de Lima Barros, PhD,* Maria Inês Fernandes Pimentel, PhD, ${ }^{*}$ Mariza de Matos Salgueiro, MD,* Rosani Santos Reis, MSc, $*$ and Armando de Oliveira Schubach, PhD*}

*FIOCRUZ, Rio de Janeiro, Brazil; and ${ }^{\dagger}$ Departamento de Otorrinolaringologia e Oftalmologia da Faculdade de Medicina, Universidade Federal do Rio de Janeiro, Rio de Janeiro, Brazil
$A^{\text {nos }}$ non immunosuppressed 73-year-old woman presented a painful ulcer, of 45 days of evolution, measuring approximately $10 \times 8 \mathrm{~cm}$, accompanied by partial destruction of the nipple and discharge of serosanguineous-purulent secretion in the left breast, simulating malignancy, surrounded by small satellite

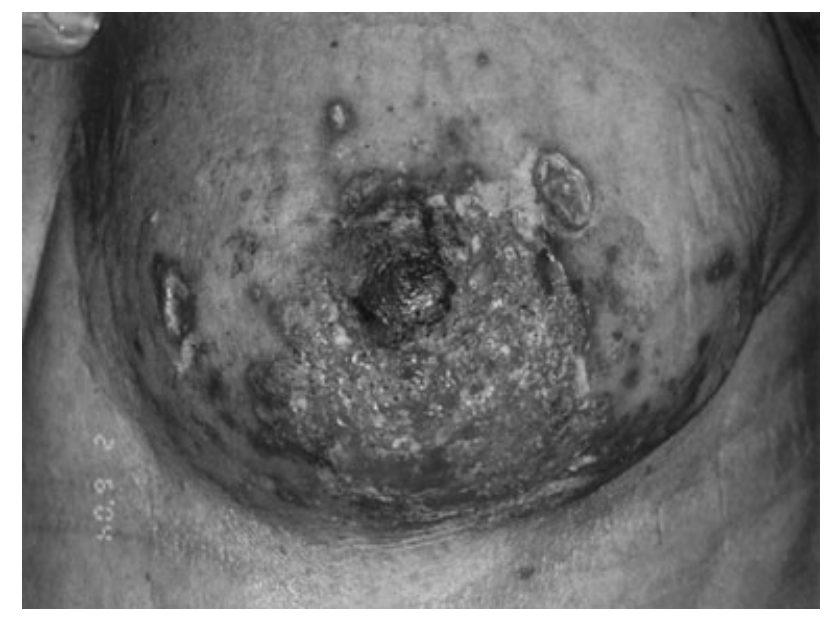

Figure 1. Ulcer, at the left breast, before treatment with itraconazole.

Address correspondence and reprint requests to: Érica de Camargo Ferreira e Vasconcellos, MSc, Fundação Oswaldo Cruz, Instituto de Pesquisa Clínica Evandro Chagas, Laboratório de Vigilância em Leishmanioses, Av. Brasil 4365, Manguinhos, Rio de Janeiro, RJ, Brazil, CEP 21040-900, or e-mail: erica.vasconcellos@ipec.fiocruz.br.

DOI: $10.1111 / j .1524-4741.2010 .00979 . x$

(๑) 2010 Wiley Periodicals, Inc., 1075-122X/10

The Breast Journal, Volume 16 Number 6, 2010 658-659 ulcers, with no lymphangitis or regional lymphadenomegaly (Fig. 1). Two mammographies were normal. She related local scratch by a cat with sporotrichosis and lived in the metropolitan region of Rio de Janeiro City, Brazil, where an epidemic of sporotrichosis transmitted by domestic cats is occurring. So, instead of performing a biopsy of the lesion at first, we seeded superficial exudate of the lesion in Sabouraud culture media. Sporothrix schenckii was isolated and treatment was started with $100 \mathrm{mg}$ itraconazole per day per os. As a good immediate therapeutic response was achieved, investigation was interrupted, without the

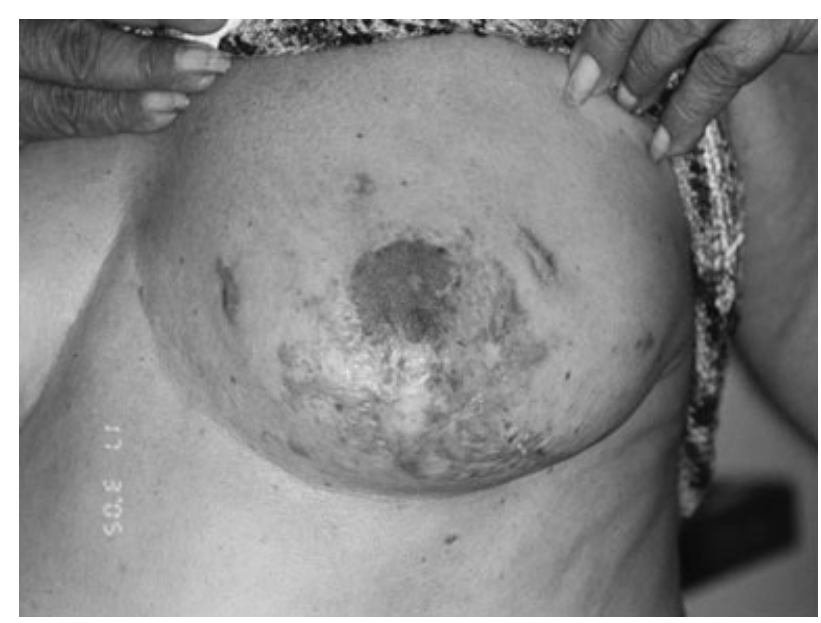

Figure 2. Ulcer's scar, at the left breast, after treatment with itraconazole. 
necessity of an anatomopathologic examination. A reduction in the diameter of the ulcer was observed after 1 month. After 7 months, the lesion was healed and presented mild erythema and desquamation, with some fibrous trabeculae in its center (Fig. 2). Itraconazole was discontinued. In the present case, the clinical and epidemiological correlation led to the correct diagnosis, confirmed by demonstration of the etiological agent.

\section{Acknowledgments}

This study was partially supported by PAPES 4 $\mathrm{CNPq} /$ Fiocruz, Brazil (Programa de Apoio à Pesquisa Estratégica 4, Conselho Nacional de Desenvolvimento Científico e Tecnológico/Fundação Oswaldo Cruz). There is no conflict of interest regarding any of the authors. 